\title{
Interactive Multiobjective Fuzzy Random Linear Programming through Fractile Criteria
}

\author{
Hitoshi Yano ${ }^{1}$ and Masatoshi Sakawa ${ }^{2}$ \\ ${ }^{1}$ School of Humanities and Social Sciences, Nagoya City University, Nagoya 467-8501, Japan \\ ${ }^{2}$ Faculty of Engineering, Hiroshima University, Hiagashi-hiroshima 739-8527, Japan \\ Correspondence should be addressed to Hitoshi Yano, yano@hum.nagoya-cu.ac.jp
}

Received 3 April 2012; Revised 24 August 2012; Accepted 19 September 2012

Academic Editor: Mariano Jimenez

Copyright (C) 2012 H. Yano and M. Sakawa. This is an open access article distributed under the Creative Commons Attribution License, which permits unrestricted use, distribution, and reproduction in any medium, provided the original work is properly cited.

\begin{abstract}
We propose an interactive fuzzy decision making method for multiobjective fuzzy random linear programming problems through fractile criteria optimization. In the proposed method, it is assumed that the decision maker has fuzzy goals for not only objective functions but also permissible probability levels in a fractile optimization model, and such fuzzy goals are quantified by eliciting the corresponding membership functions. Using the fuzzy decision, such two kinds of membership functions are integrated. In the integrated membership space, the satisfactory solution is obtained from among an extended Pareto optimal solution set through the interaction with the decision maker. An illustrative numerical example is provided to demonstrate the feasibility and efficiency of the proposed method.
\end{abstract}

\section{Introduction}

In the real world decision making situations, we often have to make a decision under uncertainty. In order to deal with decision problems involving uncertainty, stochastic programming approaches [1-4] and fuzzy programming approaches [5-7] have been developed. Recently, mathematical programming problems with fuzzy random variables [8] have been proposed [9-11] whose concept includes both probabilistic uncertainty and fuzzy one simultaneously. Extensions to multiobjective fuzzy random linear programming problems (MOFRLP) have been done and interactive methods to obtain the satisfactory solution for the decision maker have been proposed [11-13]. In their methods, it is required in advance for the decision maker to specify permissible possibility levels in a probability maximization model or permissible probability levels in a fractile optimization model. However, it seems to be very difficult for the decision maker to specify such permissible levels appropriately. From such a point of view, a fuzzy approach to MOFRLP, in which the decision maker specifies the membership functions for the fuzzy goals of both objective functions and permissible probability levels has been proposed [14]. In the proposed method, it is assumed that the decision maker adopts the fuzzy decision [6] to integrate the membership functions. However, the fuzzy decision can be viewed as one special operator to integrate the membership functions. If the decision maker would not adopt the fuzzy decision, the proposed method can not be applied in the real-world decision situation.

As one kind of the multiobjective programming problems in the real-world decision making problems, the agricultural resource management problems have been formulated and investigated to obtain satisfactory solution [15, 16]. Unfortunately, in the agricultural resource management problems, there are many kinds of uncertainty, such as future profits for crops and the various influence of the future weather. In order to deal with such uncertainty, Itoh et al. [17] formulated the agricultural resource management problem as a stochastic programming problem, and proposed a fuzzy approach to obtain the optimal cultivation areas for several kinds of vegetables. Toyonaga et al. [18] formulated the agricultural resource management problem as a fuzzy random programming problem, where the profit coefficients of the crops are defined as fuzzy random variables. 
Under these circumstances, in this paper, we propose an interactive fuzzy decision making method for MOFRLP to obtain the satisfactory solution from among an extended Pareto optimal solution set. Using a concept of a possibility measure [19], MOFRLP is transformed into multiobjective stochastic programming ones. Through a fractile optimization model, they are further transformed into usual multiobjective programming problems. An extended Pareto optimal concept is introduced and the minmax problem is formulated to obtain an extended Pareto optimal solution. An interactive algorithm is introduced to obtain the satisfactory solution from among an extended Pareto optimal solution set by solving the minmax problem on the basis of the linear programming technique. In order to illustrate the proposed method, a two-objective fuzzy random linear programming problem is formulated as a numerical example, and the interactive processes under the hypothetical decision maker are demonstrated.

\section{Multiobjective Fuzzy Random Linear Programming Problems}

Throughout this paper, assuming that coefficients of the objective functions are fuzzy random variables, we focus on the following multiobjective fuzzy random linear programming problem (MOFRLP):

[MOFRLP]

$$
\begin{array}{ll}
\text { minimize } & \widetilde{\overline{\mathbf{C}}} \mathbf{x}=\left(\widetilde{\overline{\mathbf{c}}}_{1} \mathbf{x}, \ldots, \widetilde{\overline{\mathbf{c}}}_{k} \mathbf{x}\right), \\
\text { subject to } & \mathbf{x} \in X \stackrel{\text { def }}{=}\left\{\mathbf{x} \in R^{n} \mid A \mathbf{x} \leq \mathbf{b}, \mathbf{x} \geq \mathbf{0}\right\},
\end{array}
$$

where $\mathbf{x}=\left(x_{1}, x_{2}, \ldots, x_{n}\right)^{T}$ is an $n$ dimensional decision variable column vector, $A$ is an $(m \times n)$ coefficient matrix, and $\mathbf{b}=\left(b_{1}, \ldots, b_{m}\right)^{T}$ is an $m$ dimensional column vector. $\tilde{\overline{\mathbf{c}}}_{i}=\left(\tilde{\bar{c}}_{i 1}, \ldots, \tilde{\bar{c}}_{i n}\right), i=1, \ldots, k$ are coefficient vectors of objective function $\widetilde{\bar{c}}_{i} \mathbf{x}$, whose elements are fuzzy random variables $[8,11,20]$, and the symbols “-” and “ " mean randomness and fuzziness, respectively.

In order to deal with the objective functions $\widetilde{\mathbf{c}}_{i} \mathbf{x}, i=$ $1, \ldots, k$ as a special version of a fuzzy random variable, an LR-type fuzzy random variable has been proposed [11-13]. Under the occurrence of each elementary event $\omega, \widetilde{\bar{c}}_{i j}(\omega)$ is a realization of an LR-type fuzzy random variable $\tilde{\bar{c}}_{i j}$, which is an LR fuzzy number [19] whose membership function is defined as follows:

$$
\mu_{\bar{c}_{i j}(\omega)}(s)= \begin{cases}L\left(\frac{\bar{d}_{i j}(\omega)-s}{\bar{\alpha}_{i j}(\omega)}\right) & \left(s \leq \bar{d}_{i j}(\omega) \forall \omega\right), \\ R\left(\frac{s-\bar{d}_{i j}(\omega)}{\bar{\beta}_{i j}(\omega)}\right) & \left(s>\bar{d}_{i j}(\omega) \forall \omega\right),\end{cases}
$$

where the function $L(t) \stackrel{\text { def }}{=} \max \{0, l(t)\}$ is a real-valued continuous function from $[0, \infty)$ to $[0,1]$, and $l(t)$ is a strictly decreasing continuous function satisfying $l(0)=1$ [19]. Also, $R(t) \stackrel{\text { def }}{=} \max \{0, r(t)\}$ satisfies the same conditions. $\bar{d}_{i j}, \bar{\alpha}_{i j}, \bar{\beta}_{i j}$ are random variables expressed by $\bar{d}_{i j}=d_{i j}^{1}+\bar{t}_{i} d_{i j}^{2}, \bar{\alpha}_{i j}=$ $\alpha_{i j}^{1}+\bar{t}_{i} \alpha_{i j}^{2}$, and $\bar{\beta}_{i j}=\beta_{i j}^{1}+\bar{t}_{i} \beta_{i j}^{2} . \bar{t}_{i}$ is a random variable whose distribution function is denoted by $T_{i}(\cdot)$ which is strictly increasing and continuous, and $d_{i j}^{1}, d_{i j}^{2}, \alpha_{i j}^{1}, \alpha_{i j}^{2}, \beta_{i j}^{1}, \beta_{i j}^{2}$ are constants. These parameters are set by the specialist who formulates MOFRLP (1).

Using a concept of a possibility measure [19], MOFRLP has been transformed into a multiobjective stochastic programming problem (MOSP) [11-13]. As shown in [1113], the realizations $\widetilde{\overline{\mathbf{c}}}_{i}(\omega) \mathbf{x}$ becomes an LR fuzzy number characterized by the following membership functions on the basis of the extension principle [19].

$$
\mu_{\tilde{\mathbf{c}}_{i}(\omega) \mathbf{x}}(y)= \begin{cases}L\left(\frac{\overline{\mathbf{d}}_{i}(\omega) \mathbf{x}-y}{\overline{\boldsymbol{\alpha}}_{i}(\omega) \mathbf{x}}\right) & y \leq \overline{\mathbf{d}}_{i}(\omega) \mathbf{x}, \\ R\left(\frac{y-\overline{\mathbf{d}}_{i}(\omega) \mathbf{x}}{\overline{\boldsymbol{\beta}}_{i}(\omega) \mathbf{x}}\right) & y>\overline{\mathbf{d}}_{i}(\omega) \mathbf{x} .\end{cases}
$$

For the realizations $\tilde{\overline{\mathbf{c}}}_{i}(\omega) \mathbf{x}, i=1, \ldots, k$ it is assumed that the decision maker has fuzzy goals $\widetilde{G}_{i}, i=1, \ldots, k[6]$, whose membership functions $\mu_{\widetilde{G}_{i}}(y), i=1, \ldots, k$ are continuous and strictly decreasing for minimization problems. By using a concept of a possibility measure [19], a degree of possibility [11] that the objective function value $\widetilde{\overline{\mathbf{c}}}_{i} \mathbf{x}$ satisfies the fuzzy goal $\widetilde{G}_{i}$ is expressed as follows.

$$
\begin{array}{r}
\prod_{\tilde{\mathbf{c}}_{i} \mathbf{X}}\left(\widetilde{G}_{i}\right) \stackrel{\text { def }}{=} \sup _{y} \min \left\{\mu_{\tilde{\mathbf{c}}_{i} \mathbf{X}}(y), \mu_{\widetilde{G}_{i}}(y)\right\}, \\
i=1, \ldots, k .
\end{array}
$$

Using a possibility measure, MOFRLP can be transformed into the following multiobjective stochastic programming problem (MOSP) [11-13]:

[MOSP]

$$
\underset{\mathbf{x} \in X}{\operatorname{maximize}}\left(\Pi_{\tilde{\overline{\mathbf{c}}}_{1} \mathbf{X}}\left(\widetilde{G}_{1}\right), \ldots, \Pi_{\tilde{\overline{\mathbf{c}}}_{k} \mathbf{x}}\left(\widetilde{G}_{k}\right)\right) .
$$

Through a probability maximization model and a fractile maximization model, MOSP (5) has been transformed into usual multiobjective programming problems and interactive algorithms to obtain a satisfactory solution has been proposed [11-13]. In their methods, the decision maker must specify permissible probability levels or permissible possibility levels for the objective functions in advance. However, it seems to be very difficult to specify appropriate permissible levels because they have a great influence on the objective function values or distribution function values. In the following sections, by assuming that the decision maker has fuzzy goals for permissible probability levels, we propose an interactive fuzzy decision making method for MOFRLP to obtain a satisfactory solution.

\section{Possibility-Based Fractile Optimization Model}

For the objective functions of MOSP (5), if we adopt a fractile optimization model, we can transform MOSP into 
the following multiobjective programming problem (MOP), where the decision maker specifies permissible probability levels $\hat{p}_{i}, i=1, \ldots, k$ in a subjective manner [11]:

$[\operatorname{MOP}(\hat{\mathbf{p}})]$

$$
\begin{aligned}
& \underset{\mathbf{x} \in X, h_{i} \in[0,1], i=1, \ldots, k}{\operatorname{maximize}}\left(h_{1}, \ldots, h_{k}\right), \\
& \text { subject to } \quad p_{i}\left(\mathbf{x}, h_{i}\right) \geq \hat{p}_{i}, \quad i=1, \ldots, k,
\end{aligned}
$$

where $\hat{\mathbf{p}}=\left(\hat{p}_{1}, \ldots, \hat{p}_{k}\right)$ is a vector of permissible probability levels.

Since a distribution function $T_{i}(\cdot)$ is continuous and strictly increasing, the constraints of $\operatorname{MOP}(\hat{\mathbf{p}})$ (6) can be transformed to the following form:

$$
\begin{aligned}
\hat{p}_{i} & \leq p_{i}\left(\mathbf{x}, h_{i}\right)=T_{i}\left(\frac{\mu_{\widetilde{G}_{i}^{-1}}^{-1}\left(h_{i}\right)-\left(\mathbf{d}_{i}^{1} \mathbf{x}-L^{-1}\left(h_{i}\right) \boldsymbol{\alpha}_{i}^{1} \mathbf{x}\right)}{\mathbf{d}_{i}^{2} \mathbf{x}-L^{-1}\left(h_{i}\right) \boldsymbol{\alpha}_{i}^{2} \mathbf{x}}\right), \\
& \left.\Leftrightarrow \mu_{\widetilde{G}_{i}^{-1}}^{-1} h_{i}\right) \\
& \geq\left(\mathbf{d}_{i}^{1} \mathbf{x}-L^{-1}\left(h_{i}\right) \boldsymbol{\alpha}_{i}^{1} \mathbf{x}\right)+T_{i}^{-1}\left(\hat{p}_{i}\right) \cdot\left(\mathbf{d}_{i}^{2} \mathbf{x}-L^{-1}\left(h_{i}\right) \boldsymbol{\alpha}_{i}^{2} \mathbf{x}\right) .
\end{aligned}
$$

Let us define the right-hand side of the inequality (7) as follows:

$$
\begin{aligned}
f_{i}\left(\mathbf{x}, h_{i}, \hat{p}_{i}\right) \stackrel{\text { def }}{=} & \left(\mathbf{d}_{i}^{1} \mathbf{x}-L^{-1}\left(h_{i}\right) \boldsymbol{\alpha}_{i}^{1} \mathbf{x}\right)+T_{i}^{-1}\left(\hat{p}_{i}\right) \\
& \cdot\left(\mathbf{d}_{i}^{2} \mathbf{x}-L^{-1}\left(h_{i}\right) \boldsymbol{\alpha}_{i}^{2} \mathbf{x}\right) .
\end{aligned}
$$

It is clear that $f_{i}\left(\mathbf{x}, h_{i}, \hat{p}_{i}\right)$ is strictly increasing with respect to $\hat{p}_{i}$. Then, $\operatorname{MOP}(\hat{\mathbf{p}})(6)$ can be equivalently transformed into the following form:

$[\mathrm{MOP}(\widehat{\mathbf{p}})]$

$$
\begin{aligned}
& \underset{\mathbf{x} \in X, h_{i} \in[0,1], i=1, \ldots, k}{\operatorname{maximize}}\left(h_{1}, \ldots, h_{k}\right), \\
& \text { subject to } \mu_{\widetilde{G}_{i}}\left(f_{i}\left(\mathbf{x}, h_{i}, \hat{p}_{i}\right)\right) \geq h_{i}, \quad i=1, \ldots, k .
\end{aligned}
$$

In the inequality constraints of $(9), f_{i}\left(\mathbf{x}, h_{i}, \hat{p}_{i}\right)$ is continuous and strictly increasing with respect to $h_{i}$ for any $\mathbf{x} \in X$. This means that the left-hand-side of the inequality constraints of (9) is continuous and strictly decreasing with respect to $h_{i}$ for any $\mathbf{x} \in X$. Since the right-hand-side of the inequality constraints of (9) is continuous and strictly increasing with respect to $h_{i}$, the inequality constraints of (9) must always satisfy the active condition, that is, $\mu_{\widetilde{G}_{i}}\left(f_{i}\left(\mathbf{x}, h_{i}, \hat{p}_{i}\right)\right)=h_{i}, i=$ $1, \ldots, k$ at the optimal solution. From such a point of view, $\operatorname{MOP}(\hat{\mathbf{p}})(9)$ is equivalently expressed as the following form:

$[\mathrm{MOP}(\hat{\mathbf{p}})]$

$$
\begin{gathered}
\underset{\mathbf{x} \in X, h_{i} \in[0,1], i=1, \ldots, k}{\operatorname{maximize}} \quad\left(\mu_{\widetilde{G}_{1}}\left(f_{1}\left(\mathbf{x}, h_{1}, \hat{p}_{1}\right)\right), \ldots,\right. \\
\left.\mu_{\widetilde{G}_{k}}\left(f_{k}\left(\mathbf{x}, h_{k}, \hat{p}_{k}\right)\right)\right), \\
\text { subject to } \quad \mu_{\widetilde{G}_{i}}\left(f_{i}\left(\mathbf{x}, h_{i}, \hat{p}_{i}\right)\right)=h_{i}, \quad i=1, \ldots, k .
\end{gathered}
$$

In MOP (10), we can define Pareto optimal solutions, which depend on the permissible probability levels $\hat{p}_{i}, i=1, \ldots, k$.

Definition 1. For the given permissible probability levels $\hat{p}_{i}, i=1, \ldots, k, \mathbf{x}^{*} \in X, h_{i}^{*} \in[0,1], i=1, \ldots, k$ is said

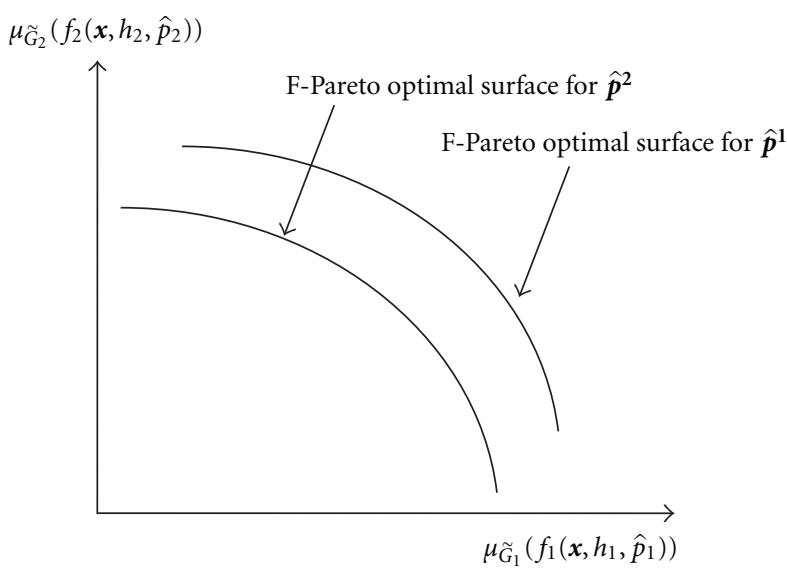

FIgURE 1: F-Pareto optimal solution sets for $\hat{\mathbf{p}}^{1} \leq \hat{\mathbf{p}}^{2}$.

to be a F-Pareto optimal solution to MOP (10), if and only if there does not exist another $\mathbf{x} \in X, h_{i} \in[0,1], i=$ $1, \ldots, k$ such that $\mu_{D_{G_{i}}}\left(\mathbf{x}, h_{i}, \hat{p}_{i}\right) \geq \mu_{D_{G_{i}}}\left(\mathbf{x}^{*}, h_{i}^{*}, \hat{p}_{i}\right), i=$ $1, \ldots, k$ with strict inequality holding for at least one $i$, where $\mu_{\widetilde{G}_{i}}\left(f_{i}\left(\mathbf{x}^{*}, h_{i}^{*}, \hat{p}_{i}\right)\right)=h_{i}^{*}, \mu_{\widetilde{G}_{i}}\left(f_{i}\left(\mathbf{x}, h_{i}, \hat{p}_{i}\right)\right)=h_{i}, i=1, \ldots, k$.

Katagiri et al. [21] have proposed an interactive decision making method for MOP (10) to obtain a satisfactory solution from among an F-Pareto optimal solution set. However, in their proposed method, the decision maker must specify permissible probability levels $\hat{\mathbf{p}}$ in advance. From the inequalities in MOP (9), it is obvious that the less values of permissible probability levels $\hat{p}_{i}, i=1, \ldots, k$ give the larger values of membership functions $\mu_{\widetilde{G}_{i}}(y), i=1, \ldots, k$. Figure 1 shows the image of F-Pareto optimal solutions for two kinds of permissible probability levels $\hat{\mathbf{p}}^{1}=\left(\hat{p}_{1}^{1}, \hat{p}_{2}^{1}\right)$ and $\hat{\mathbf{p}}^{2}=\left(\hat{p}_{1}^{2}, \hat{p}_{2}^{2}\right)$, where $\hat{p}_{i}^{1} \leq \hat{p}_{i}^{2}, i=1,2$. From Figure 1 , there exists an infinite number of Pareto optimal solution sets corresponding to permissible probability levels. In the interactive decision making method proposed by Katagiri et al. [21], it is assumed that the decision maker can specify appropriate permissible probability levels. However, because of a conflict between permissible probability levels $\hat{p}_{i}, i=$ $1, \ldots, k$ and the corresponding values of the membership functions $\mu_{\widetilde{G}_{i}}(y), i=1, \ldots, k$, it seems to be difficult for the decision maker to specify permissible probability levels appropriately. In general, the decision maker seems to prefer not only the larger value of a permissible probability level but also the larger value of the corresponding membership functions $\mu_{\tilde{G}_{i}}(\cdot)$. From such a point of view, we consider the following multiobjective programming problem which can be regarded as a natural extension of $\operatorname{MOP}(\hat{\mathbf{p}})$ :

[MOP]

$$
\begin{aligned}
\underset{x \in X, h_{i} \in[0,1], \hat{p}_{i} \in(0,1), i=1, \ldots, k}{\operatorname{maximize}}( & \mu_{\widetilde{G}_{1}}\left(f_{1}\left(\mathbf{x}, h_{1}, \hat{p}_{1}\right)\right), \ldots, \\
& \left.\mu_{\widetilde{G}_{k}}\left(f_{k}\left(\mathbf{x}, h_{k}, \hat{p}_{k}\right)\right), \hat{p}_{1}, \ldots, \hat{p}_{k}\right),
\end{aligned}
$$

subject to $\mu_{\widetilde{G}_{i}}\left(f_{i}\left(\mathbf{x}, h_{i}, \hat{p}_{i}\right)\right)=h_{i}, \quad i=1, \ldots, k$. 
It should be noted in MOP that permissible probability levels are not the fixed values but the decision variables.

Considering the imprecise nature of the decision maker's judgment, we assume that the decision maker has a fuzzy goal for each permissible probability level. Such a fuzzy goal can be quantified by eliciting the corresponding membership function. Let us denote a membership function of a permissible probability level $\hat{p}_{i}$ as $\mu_{\hat{p}_{i}}\left(\hat{p}_{i}\right)$. Then, MOP can be transformed as the following multiobjective programming problem:

[MOP]

$$
\begin{aligned}
& \underset{\mathbf{x} \in X, h_{i} \in[0,1], \hat{p}_{i} \in(0,1), i=1, \ldots, k}{\operatorname{maximize}} \quad\left(\mu_{\widetilde{G}_{1}}\left(f_{1}\left(\mathbf{x}, h_{1}, \hat{p}_{1}\right)\right), \ldots,\right. \\
& \mu_{\widetilde{G}_{k}}\left(f_{k}\left(\mathbf{x}, h_{k}, \hat{p}_{k}\right)\right), \\
& \left.\mu_{\hat{p}_{1}}\left(\hat{p}_{1}\right), \ldots, \mu_{\hat{p}_{k}}\left(\hat{p}_{k}\right)\right),
\end{aligned}
$$

subject to $\mu_{\widetilde{G}_{i}}\left(f_{i}\left(\mathbf{x}, h_{i}, \hat{p}_{i}\right)\right)=h_{i}, \quad i=1, \ldots, k$.

In order to elicit the membership functions appropriately, we suggest the following procedures. First of all, the decision maker sets the intervals $P_{i}=\left[p_{i \min }, p_{i \max }\right], i=1, \ldots, k$, where $p_{i \text { min }}$ is an unacceptable maximum value of $\hat{p}_{i}$ and $p_{i \max }$ is a sufficiently satisfactory minimum value of $\hat{p}_{i}$. Throughout this section, we make the following assumption.

Assumption 1. $\mu_{\hat{p}_{i}}\left(\hat{p}_{i}\right), i=1, \ldots, k$ are strictly increasing and continuous with respect to $\hat{p}_{i} \in P_{i}$, and $\mu_{\hat{p}_{i}}\left(p_{i \text { min }}\right)=0$, $\mu_{\hat{p}_{i}}\left(p_{i \max }\right)=1$.

Corresponding to the interval $P_{i}$, the interval of $h_{i}$, which is defined as $H_{i}\left(P_{i}\right)=\left[h_{i \min }, h_{i \max }\right]$, can be obtained as follows. The maximum value $h_{i \max }$ can be obtained by solving the following problem:

$$
\begin{array}{ll}
\underset{\mathbf{x} \in X, h_{i} \in[0,1]}{\operatorname{minimize}} & f_{i}\left(\mathbf{x}, h_{i}, p_{i \min }\right) \\
\text { subject to } & h_{i}=\mu_{\widetilde{G}_{i}}\left(f_{i}\left(\mathbf{x}, h_{i}, p_{i \min }\right)\right)
\end{array}
$$

This is equivalent to the following problem:

$$
\begin{aligned}
h_{i \max } \stackrel{\text { def }}{=} \underset{\mathbf{x} \in X, h_{i} \in[0,1]}{\operatorname{maximize}} h_{i}, & \\
\text { subject to } \quad & \begin{aligned}
\mu_{\widetilde{G}_{i}}^{-1}\left(h_{i}\right)=\left(\mathbf{d}_{i}^{1} \mathbf{x}-L^{-1}\left(h_{i}\right) \boldsymbol{\alpha}_{i}^{1} \mathbf{x}\right) \\
\\
\quad+T_{i}^{-1}\left(p_{i \min }\right) \cdot\left(\mathbf{d}_{i}^{2} \mathbf{x}-L^{-1}\left(h_{i}\right) \boldsymbol{\alpha}_{i}^{2} \mathbf{x}\right) .
\end{aligned}
\end{aligned}
$$

The optimal solution $\mathbf{x}^{*}, h_{i}^{*}, i=1, \ldots, k$ of the above problem can be obtained by combined use of the bisection method with respect to $h_{i} \in[0,1]$ and the first-phase of the two-phase simplex method of linear programming. In order to obtain $h_{i \min }$, we first solve the following $k$ linear programming problems:

$$
\begin{aligned}
& \underset{\mathbf{x} \in X, h_{i} \in[0,1]}{\operatorname{minimize}} \quad f_{i}\left(\mathbf{x}, h_{i}, p_{i \max }\right), \\
& \text { subject to } \quad h_{i}=\mu_{\widetilde{G}_{i}}\left(f_{i}\left(\mathbf{x}, h_{i}, p_{i \max }\right)\right) .
\end{aligned}
$$

Let $\left(\mathbf{x}_{i}^{*}, h_{i}^{*}\right), i=1, \ldots, k$ be the above optimal solution. Using the optimal solutions $\left(\mathbf{x}_{i}^{*}, h_{i}^{*}\right), i=1, \ldots, k, h_{i \min }$ can be obtained as follows:

$$
h_{i \min } \stackrel{\text { def }}{=} \min _{\ell=1, \ldots, k, \ell \neq i} \mu_{\widetilde{G}_{i}}\left(f_{i}\left(\mathbf{x}_{\ell}^{*}, h_{\ell}^{*}, p_{i \max }\right)\right) .
$$

It should be noted here that, $\mu_{\widetilde{G}_{i}}\left(f_{i}\left(\mathbf{x}, h_{i}, \hat{p}_{i}\right)\right)$ is strictly decreasing with respect to $\hat{p}_{i}$. If the decision maker adopts the fuzzy decision [6] to integrate $\mu_{\widetilde{G}_{i}}\left(f_{i}\left(\mathbf{x}, h_{i}, \hat{p}_{i}\right)\right)$ and $\mu_{\hat{p}_{i}}\left(\hat{p}_{i}\right)$, MOP can be transformed into the following form:

[MOP]

$$
\begin{aligned}
& \underset{\mathbf{x} \in X, \hat{p}_{i} \in P_{i}, h_{i} \in H_{i}\left(P_{i}\right), i=1, \ldots, k}{\operatorname{maximize}} \quad\left(\mu_{D_{G_{1}}}\left(\mathbf{x}, h_{1}, \hat{p}_{1}\right), \ldots,\right. \\
& \left.\mu_{D_{G_{k}}}\left(\mathbf{x}, h_{k}, \hat{p}_{k}\right)\right),
\end{aligned}
$$

subject to $\mu_{\widetilde{G}_{i}}\left(f_{i}\left(\mathbf{x}, h_{i}, \hat{p}_{i}\right)\right)=h_{i}, \quad i=1, \ldots, k$,

where

$$
\mu_{D_{G_{i}}}\left(\mathbf{x}, h_{i}, \hat{p}_{i}\right) \stackrel{\text { def }}{=} \min \left\{\mu_{\hat{p}_{i}}\left(\hat{p}_{i}\right), \mu_{\widetilde{G}_{i}}\left(f_{i}\left(\mathbf{x}, h_{i}, \hat{p}_{i}\right)\right)\right\}
$$

In order to deal with MOP (17), we introduce the following concept of an $M_{P F}$-Pareto optimal solution, which can be viewed as an M-Pareto optimal solution through possibilitybased fractile models.

Definition 2. $\mathbf{x}^{*} \in X, \hat{p}_{i}^{*} \in P_{i}, h_{i}^{*} \in H_{i}\left(P_{i}\right), i=1, \ldots, k$ is said to be an $M_{P F}$-Pareto optimal solution to MOP (17), if and only if there does not exist another $\mathbf{x} \in X, \hat{p}_{i} \in$ $P_{i}, h_{i} \in H_{i}\left(P_{i}\right), i=1, \ldots, k$ such that $\mu_{D_{G_{i}}}\left(\mathbf{x}, h_{i}, \hat{p}_{i}\right) \geq$ $\mu_{D_{G_{i}}}\left(\mathbf{x}^{*}, h_{i}^{*}, \hat{p}_{i}^{*}\right), i=1, \ldots, k$ with strict inequality holding for at least one $i$, where $\mu_{\widetilde{G}_{i}}\left(f_{i}\left(\mathbf{x}^{*}, h_{i}^{*}, \hat{p}_{i}^{*}\right)\right)=h_{i}^{*}$, $\mu_{\widetilde{G}_{i}}\left(f_{i}\left(\mathbf{x}, h_{i}, \hat{p}_{i}\right)\right)=h_{i}, i=1, \ldots, k$.

For generating a candidate of a satisfactory solution which is also $M_{P F}$-Pareto optimal, the decision maker is asked to specify the reference membership values [6]. Once the reference membership values $\hat{\mu}=\left(\hat{\mu}_{1}, \ldots, \hat{\mu}_{k}\right)$ are specified, the corresponding $M_{P F}$-Pareto optimal solution is obtained by solving the following minmax problem:

$[\operatorname{MINMAX}(\hat{\boldsymbol{\mu}})]$

$$
\begin{array}{cl}
\underset{\mathbf{x} \in X, \hat{p}_{i} \in P_{i}, h_{i} \in H_{i}\left(P_{i}\right), i=1, \ldots, k, \lambda \in \Lambda}{\operatorname{minimize}} \lambda, \\
\text { subject to } & \hat{\mu}_{i}-\mu_{\hat{p}_{i}}\left(\hat{p}_{i}\right) \leq \lambda, \quad i=1, \ldots, k, \\
& \hat{\mu}_{i}-h_{i} \leq \lambda, \quad i=1, \ldots, k, \\
& \mu_{\widetilde{G}_{i}}\left(f_{i}\left(\mathbf{x}, h_{i}, \hat{p}_{i}\right)\right)=h_{i}, \quad i=1, \ldots, k,
\end{array}
$$

where

$$
\Lambda=\left[\max _{i=1, \ldots, k} \hat{\mu}_{i}-1, \min _{i=1, \ldots, k} \hat{\mu}_{i}\right] .
$$


In the constraints of (19), it holds that

$$
\begin{aligned}
h_{i}= & \mu_{\widetilde{G}_{i}}\left(f_{i}\left(\mathbf{x}, h_{i}, \hat{p}_{i}\right)\right) \geq \hat{\mu}_{i}-\lambda, \\
\Leftrightarrow & \mu_{\widetilde{G}_{i}}^{-1}\left(h_{i}\right)=f_{i}\left(\mathbf{x}, h_{i}, \hat{p}_{i}\right) \leq \mu_{\widetilde{G}_{i}}^{-1}\left(\hat{\mu}_{i}-\lambda\right) \\
\Longleftrightarrow & \mu_{\widetilde{G}_{i}}^{-1}\left(h_{i}\right)=\left(\mathbf{d}_{i}^{1} \mathbf{x}-L^{-1}\left(h_{i}\right) \boldsymbol{\alpha}_{i}^{1} \mathbf{x}\right) \\
& +T_{i}^{-1}\left(\hat{p}_{i}\right) \cdot\left(\mathbf{d}_{i}^{2} \mathbf{x}-L^{-1}\left(h_{i}\right) \boldsymbol{\alpha}_{i}^{2} \mathbf{x}\right) \\
\leq & \mu_{\widetilde{G}_{i}}^{-1}\left(\hat{\mu}_{i}-\lambda\right) .
\end{aligned}
$$

In the right hand side of $(21)$, because of $L^{-1}\left(h_{i}\right) \leq L^{-1}\left(\hat{\mu}_{i}-\right.$ $\lambda)$ and $\boldsymbol{\alpha}_{i}^{1} \mathbf{x}+T_{i}^{-1}\left(\hat{p}_{i}\right) \boldsymbol{\alpha}_{i}^{2} \mathbf{x}>0$, it holds that

$$
\begin{aligned}
\left(\mathbf{d}_{i}^{1} \mathbf{x}\right. & \left.-L^{-1}\left(h_{i}\right) \boldsymbol{\alpha}_{i}^{1} \mathbf{x}\right)+T_{i}^{-1}\left(\hat{p}_{i}\right) \cdot\left(\mathbf{d}_{i}^{2} \mathbf{x}-L^{-1}\left(h_{i}\right) \boldsymbol{\alpha}_{i}^{2} \mathbf{x}\right) \\
= & \left(\mathbf{d}_{i}^{1} \mathbf{x}+T_{i}^{-1}\left(\hat{p}_{i}\right) \mathbf{d}_{i}^{2} \mathbf{x}\right)-L^{-1}\left(h_{i}\right)\left(\boldsymbol{\alpha}_{i}^{1} \mathbf{x}+T_{i}^{-1}\left(\hat{p}_{i}\right) \boldsymbol{\alpha}_{i}^{2} \mathbf{x}\right) \\
\geq & \left(\mathbf{d}_{i}^{1} \mathbf{x}+T_{i}^{-1}\left(\hat{p}_{i}\right) \mathbf{d}_{i}^{2} \mathbf{x}\right)-L^{-1}\left(\hat{\mu}_{i}-\lambda\right) \\
& \times\left(\boldsymbol{\alpha}_{i}^{1} \mathbf{x}+T_{i}^{-1}\left(\hat{p}_{i}\right) \boldsymbol{\alpha}_{i}^{2} \mathbf{x}\right) .
\end{aligned}
$$

Using (21) and (22), it holds that

$$
\begin{aligned}
\mu_{\widetilde{G}_{i}}^{-1}\left(\hat{\mu}_{i}-\lambda\right) \\
\geq \\
=\left(\mathbf{d}_{i}^{1} \mathbf{x}+T_{i}^{-1}\left(\hat{p}_{i}\right) \mathbf{d}_{i}^{2} \mathbf{x}\right)-L^{-1}\left(\hat{\mu}_{i}-\lambda\right)\left(\boldsymbol{\alpha}_{i}^{1} \mathbf{x}+T_{i}^{-1}\left(\hat{p}_{i}\right) \boldsymbol{\alpha}_{i}^{2} \mathbf{x}\right) \\
=\left(\mathbf{d}_{i}^{1} \mathbf{x}-L^{-1}\left(\hat{\mu}_{i}-\lambda\right) \boldsymbol{\alpha}_{i}^{1} \mathbf{x}\right)+T_{i}^{-1}\left(\hat{p}_{i}\right) \\
\quad \cdot\left(\mathbf{d}_{i}^{2} \mathbf{x}-L^{-1}\left(\hat{\mu}_{i}-\lambda\right) \boldsymbol{\alpha}_{i}^{2} \mathbf{x}\right) .
\end{aligned}
$$

From the inequality $\hat{\mu}_{i}-\mu_{\hat{p}_{i}}\left(\hat{p}_{i}\right) \leq \lambda$ in (19) and Assumption 1, it holds that $\hat{p}_{i} \geq \mu_{\hat{p}_{i}}^{-1}\left(\hat{\mu}_{i}-\lambda\right)$. Therefore, (23) can be transformed into the following form:

$$
\begin{aligned}
& T_{i}\left(\frac{\mu_{\widetilde{G}_{i}}^{-1}\left(\hat{\mu}_{i}-\lambda\right)-\left(\mathbf{d}_{i}^{1} \mathbf{x}-L^{-1}\left(\hat{\mu}_{i}-\lambda\right) \boldsymbol{\alpha}_{i}^{1} \mathbf{x}\right)}{\mathbf{d}_{i}^{2} \mathbf{x}-L^{-1}\left(\hat{\mu}_{i}-\lambda\right) \boldsymbol{\alpha}_{i}^{2} \mathbf{x}}\right) \\
& \geq \hat{p}_{i} \geq \mu_{\hat{p}_{i}}^{-1}\left(\hat{\mu}_{i}-\lambda\right), \\
& \Leftrightarrow \mu_{\widetilde{G}_{i}}^{-1}\left(\hat{\mu}_{i}-\lambda\right) \geq\left(\mathbf{d}_{i}^{1} \mathbf{x}-L^{-1}\left(\hat{\mu}_{i}-\lambda\right) \boldsymbol{\alpha}_{i}^{1} \mathbf{x}\right) \\
& \quad+T_{i}^{-1}\left(\mu_{\hat{p}_{i}}^{-1}\left(\hat{\mu}_{i}-\lambda\right)\right) \cdot\left(\mathbf{d}_{i}^{2} \mathbf{x}-L^{-1}\left(\hat{\mu}_{i}-\lambda\right) \boldsymbol{\alpha}_{i}^{2} \mathbf{x}\right) .
\end{aligned}
$$

Therefore, MINMAX (19) can be reduced to the following minmax problem:

$[\operatorname{MINMAX}(\hat{\boldsymbol{\mu}})]$

$$
\begin{array}{ll}
\underset{\mathbf{x} \in X, \lambda \in \Lambda}{\operatorname{minimize}} & \lambda \\
\text { subject to } & \mu_{\widetilde{G}_{i}}^{-1}\left(\widehat{\mu}_{i}-\lambda\right) \geq\left(\mathbf{d}_{i}^{1} \mathbf{x}-L^{-1}\left(\hat{\mu}_{i}-\lambda\right) \boldsymbol{\alpha}_{i}^{1} \mathbf{x}\right) \\
& +T_{i}^{-1}\left(\mu_{\hat{p}_{i}}^{-1}\left(\hat{\mu}_{i}-\lambda\right)\right) \cdot\left(\mathbf{d}_{i}^{2} \mathbf{x}-L^{-1}\left(\hat{\mu}_{i}-\lambda\right) \boldsymbol{\alpha}_{i}^{2} \mathbf{x}\right), \\
& i=1, \ldots, k .
\end{array}
$$

The relationships between the optimal solution $\left(\mathbf{x}^{*}, \lambda^{*}\right)$ of $\operatorname{MINMAX}(\hat{\boldsymbol{\mu}})(25)$ and $M_{P F}$-Pareto optimal solutions can be characterized by the following theorem.

\section{Theorem 3.}

(1) If $\mathbf{x}^{*} \in X, \lambda^{*} \in \Lambda$ is a unique optimal solution of $\operatorname{MINMAX}(\hat{\boldsymbol{\mu}})(25)$, then $\mathbf{x}^{*}, \hat{p}_{i}^{*}=\mu_{\hat{p}_{i}}^{-1}\left(\hat{\mu}_{i}-\lambda^{*}\right), h_{i}^{*}=$ $\hat{\mu}_{i}-\lambda^{*}, i=1, \ldots, k$ is an $M_{P F}$-Pareto optimal solution.

(2) If $\mathbf{x}^{*} \in X, \hat{p}_{i}^{*} \in P_{i}, h_{i}^{*} \in H_{i}\left(P_{i}\right), i=1, \ldots, k$ is an $M_{P F}$-Pareto optimal solution, then $\mathbf{x}^{*}, \lambda^{*}=\hat{\mu}_{i}-\mu_{\hat{p}_{i}}\left(\hat{p}_{i}^{*}\right)=$ $\hat{\mu}_{i}-\mu_{\widetilde{G}_{i}}\left(f_{i}\left(\mathbf{x}^{*}, h_{i}^{*}, \hat{p}_{i}^{*}\right)\right), i=1, \ldots, k$ is an optimal solution of $\operatorname{MINMAX}(\hat{\boldsymbol{\mu}})(25)$ for some reference membership values $\hat{\boldsymbol{\mu}}=$ $\left(\hat{\mu}_{1}, \ldots, \hat{\mu}_{k}\right)$.

Proof. (1) From the inequality constraints of (25), it holds that

$$
\hat{\mu}_{i}-\lambda^{*} \leq \mu_{\widetilde{G}_{i}}\left(f_{i}\left(\mathbf{x}^{*}, \hat{\mu}_{i}-\lambda^{*}, \mu_{\hat{p}_{i}}^{-1}\left(\hat{\mu}_{i}-\lambda^{*}\right)\right)\right),
$$

and it is obvious that $\hat{\mu}_{i}-\lambda^{*}=\mu_{\hat{p}_{i}}\left(\mu_{\hat{p}_{i}}^{-1}\left(\hat{\mu}_{i}-\lambda^{*}\right)\right)$. Assume that $\mathbf{x}^{*} \in X, \hat{\mu}_{i}-\lambda^{*} \in H_{i}\left(P_{i}\right), \mu_{\hat{p}_{i}}^{-1}\left(\hat{\mu}_{i}-\lambda^{*}\right) \in P_{i}, i=1, \ldots, k$ is not an $M_{P F}$-Pareto optimal solution. Then, there exist $\mathbf{x} \in$ $X, \hat{p}_{i} \in P_{i}, h_{i} \in H_{i}\left(P_{i}\right), i=1, \ldots, k$ such that

$$
\begin{aligned}
\mu_{D_{G_{i}}}\left(\mathbf{x}, h_{i}, \hat{p}_{i}\right) & =\min \left\{\mu_{\hat{p}_{i}}\left(\hat{p}_{i}\right), \mu_{\widetilde{G}_{i}}\left(f_{i}\left(\mathbf{x}, h_{i}, \hat{p}_{i}\right)\right)\right\} \\
& \geq \mu_{D_{G_{i}}}\left(\mathbf{x}^{*}, \hat{\mu}_{i}-\lambda^{*}, \mu_{\hat{p}_{i}}^{-1}\left(\hat{\mu}_{i}-\lambda^{*}\right)\right) \\
& =\hat{\mu}_{i}-\lambda^{*}, \quad i=1, \ldots, k,
\end{aligned}
$$

with strict inequality holding for at least one $i$, and $\mu_{\widetilde{G}_{i}}\left(f_{i}\left(\mathbf{x}, h_{i}, \hat{p}_{i}\right)\right)=h_{i}, i=1, \ldots, k$. Then it holds that

$$
\begin{gathered}
\mu_{\hat{p}_{i}}\left(\hat{p}_{i}\right) \geq \hat{\mu}_{i}-\lambda^{*}, \quad i=1, \ldots, k, \\
\mu_{\widetilde{G}_{i}}\left(f_{i}\left(\mathbf{x}, h_{i}, \hat{p}_{i}\right)\right) \geq \hat{\mu}_{i}-\lambda^{*}, \quad i=1, \ldots, k .
\end{gathered}
$$

From Assumption 1 and (8), (28) can be transformed as follows:

$$
\begin{array}{r}
\hat{p}_{i} \geq \mu_{\hat{p}_{i}}^{-1}\left(\hat{\mu}_{i}-\lambda^{*}\right), \quad i=1, \ldots, k, \\
\hat{p}_{i} \leq T_{i}\left(\frac{\mu_{\widetilde{G}_{i}}^{-1}\left(\hat{\mu}_{i}-\lambda^{*}\right)-\left(\mathbf{d}_{i}^{1} \mathbf{x}-L^{-1}\left(h_{i}\right) \boldsymbol{\alpha}_{i}^{1} \mathbf{x}\right)}{\mathbf{d}_{i}^{2} \mathbf{x}-L^{-1}\left(h_{i}\right) \boldsymbol{\alpha}_{i}^{2} \mathbf{x}}\right), \\
i=1, \ldots, k .
\end{array}
$$


Because of $L^{-1}\left(h_{i}\right) \leq L^{-1}\left(\widehat{\mu}_{i}-\lambda^{*}\right), i=1, \ldots, k$, there exists $\mathbf{x} \in X$ such that

$$
\begin{aligned}
\mu_{\widetilde{G}_{i}}^{-1}\left(\hat{\mu}_{i}-\lambda^{*}\right)-\left(\mathbf{d}_{i}^{1} \mathbf{x}-L^{-1}\left(h_{i}\right) \boldsymbol{\alpha}_{i}^{1} \mathbf{x}\right) \\
\geq T_{i}^{-1}\left(\mu_{\hat{p}_{i}}^{-1}\left(\hat{\mu}_{i}-\lambda^{*}\right)\right) \cdot\left(\mathbf{d}_{i}^{2} \mathbf{x}-L^{-1}\left(h_{i}\right) \boldsymbol{\alpha}_{i}^{2} \mathbf{x}\right), \\
\Longleftrightarrow \mu_{\widetilde{G}_{i}}^{-1}\left(\hat{\mu}_{i}-\lambda^{*}\right) \\
\geq\left(\mathbf{d}_{i}^{1} \mathbf{x}+T_{i}^{-1}\left(\mu_{\hat{p}_{i}}^{-1}\left(\hat{\mu}_{i}-\lambda^{*}\right)\right) \cdot \mathbf{d}_{i}^{2} \mathbf{x}\right) \\
\quad-L^{-1}\left(h_{i}\right)\left(\boldsymbol{\alpha}_{i}^{1} \mathbf{x}+T_{i}^{-1}\left(\mu_{\hat{p}_{i}}^{-1}\left(\hat{\mu}_{i}-\lambda^{*}\right)\right) \cdot \boldsymbol{\alpha}_{i}^{2} \mathbf{x}\right), \\
\Longleftrightarrow \mu_{\widetilde{G}_{i}}^{-1}\left(\hat{\mu}_{i}-\lambda^{*}\right) \geq\left(\mathbf{d}_{i}^{1} \mathbf{x}+T_{i}^{-1}\left(\mu_{\hat{p}_{i}}^{-1}\left(\hat{\mu}_{i}-\lambda^{*}\right)\right) \cdot \mathbf{d}_{i}^{2} \mathbf{x}\right) \\
\quad-L^{-1}\left(\hat{\mu}_{i}-\lambda^{*}\right)\left(\boldsymbol{\alpha}_{i}^{1} \mathbf{x}+T_{i}^{-1}\left(\mu_{\hat{p}_{i}}^{-1}\left(\hat{\mu}_{i}-\lambda^{*}\right)\right) \cdot \boldsymbol{\alpha}_{i}^{2} \mathbf{x}\right),
\end{aligned}
$$

This contradicts the fact that $\mathbf{x}^{*} \in X, \lambda^{*} \in \Lambda$ is a unique optimal solution to $\operatorname{MINMAX}(\hat{\boldsymbol{\mu}})(25)$.

(2) Assume that $\mathbf{x}^{*} \in X, \lambda^{*} \in \Lambda$ is not an optimal solution to $\operatorname{MINMAX}(\hat{\boldsymbol{\mu}})$ (25) for any reference membership values $\hat{\boldsymbol{\mu}}=\left(\hat{\mu}_{1}, \ldots, \hat{\mu}_{k}\right)$ which satisfy the equalities

$$
\hat{\mu}_{i}-\lambda^{*}=\mu_{\hat{p}_{i}}\left(\hat{p}_{i}^{*}\right)=\mu_{\widetilde{G}_{i}}\left(f_{i}\left(\mathbf{x}^{*}, h_{i}^{*}, \hat{p}_{i}^{*}\right)\right), \quad i=1, \ldots, k .
$$

Then, there exists some $\mathbf{x} \in X, \lambda<\lambda^{*}$ such that

$$
\begin{array}{r}
\mu_{\widetilde{G}_{i}}^{-1}\left(\hat{\mu}_{i}-\lambda\right)-\left(\mathbf{d}_{i}^{1} \mathbf{x}-L^{-1}\left(\hat{\mu}_{i}-\lambda\right) \boldsymbol{\alpha}_{i}^{1} \mathbf{x}\right) \\
\geq T_{i}^{-1}\left(\mu_{\hat{p}_{i}}^{-1}\left(\hat{\mu}_{i}-\lambda\right)\right) \cdot\left(\mathbf{d}_{i}^{2} \mathbf{x}-L^{-1}\left(\hat{\mu}_{i}-\lambda\right) \boldsymbol{\alpha}_{i}^{2} \mathbf{x}\right), \\
\Longleftrightarrow \mu_{\widetilde{G}_{i}}\left(f_{i}\left(\mathbf{x}, \hat{\mu}_{i}-\lambda, \mu_{\hat{p}_{i}}^{-1}\left(\hat{\mu}_{i}-\lambda\right)\right)\right) \geq \hat{\mu}_{i}-\lambda, \\
i=1, \ldots, k,
\end{array}
$$

Because of (31), (32) and $\hat{\mu}_{i}-\lambda>\hat{\mu}_{i}-\lambda^{*}, i=1, \ldots, k$, the following inequalities hold:

$$
\begin{gathered}
\mu_{\hat{p}_{i}}\left(\hat{p}_{i}\right)>\mu_{\hat{p}_{i}}\left(\hat{p}_{i}^{*}\right), \quad i=1, \ldots, k, \\
\mu_{\widetilde{G}_{i}}\left(f_{i}\left(\mathbf{x}, \hat{h}_{i}, \hat{p}_{i}\right)\right)>\mu_{\widetilde{G}_{i}}\left(f_{i}\left(\mathbf{x}^{*}, h_{i}^{*}, \hat{p}_{i}^{*}\right)\right), \quad i=1, \ldots, k,
\end{gathered}
$$

where $\hat{p}_{i}=\mu_{\hat{p}_{i}}^{-1}\left(\mu_{i}-\lambda\right) \in P_{i}, \hat{h}_{i}=\hat{\mu}_{i}-\lambda \in H_{i}\left(P_{i}\right), i=$ $1, \ldots, k$. This means that there exists some $\mathbf{x} \in X, \hat{p}_{i} \in$ $P_{i}, \hat{h}_{i} \in H_{i}\left(P_{i}\right), i=1, \ldots, k$ such that $\mu_{D_{G_{i}}}\left(\mathbf{x}, \hat{h}_{i}, \hat{p}_{i}\right)>$ $\mu_{D_{G_{i}}}\left(\mathbf{x}^{*}, h_{i}^{*}, \hat{p}_{i}^{*}\right), i=1, \ldots, k$. This contradicts the fact that $\mathbf{x}^{*} \in X, \hat{p}_{i}^{*} \in P_{i}, h_{i}^{*} \in H_{i}\left(P_{i}\right), i=1, \ldots, k$ is an $M_{P F}$-Pareto optimal solution.

\section{Pareto Optimality Test}

From Theorem 3, it is not guaranteed that the optimal solution $\left(\mathbf{x}^{*}, \lambda^{*}\right)$ of $\operatorname{MINMAX}(\hat{\boldsymbol{\mu}})(25)$ is $M_{P F}$-Pareto optimal, if it is not unique. In order to guarantee the $M_{P F}$-Pareto optimality, we first assume that $k$ constraints of $\operatorname{MINMAX}(\hat{\boldsymbol{\mu}})$ (25) are active at the optimal solution $\left(\mathbf{x}^{*}, \lambda^{*}\right)$, that is,

$$
\begin{array}{r}
\mu_{\widetilde{G}_{i}}^{-1}\left(\hat{\mu}_{i}-\lambda^{*}\right)-\left(\mathbf{d}_{i}^{1} \mathbf{x}^{*}-L^{-1}\left(\hat{\mu}_{i}-\lambda^{*}\right) \boldsymbol{\alpha}_{i}^{1} \mathbf{x}^{*}\right) \\
=T_{i}^{-1}\left(\mu_{\hat{p}_{i}}^{-1}\left(\hat{\mu}_{i}-\lambda^{*}\right)\right) \cdot\left(\mathbf{d}_{i}^{2} \mathbf{x}^{*}-L^{-1}\left(\hat{\mu}_{i}-\lambda^{*}\right) \boldsymbol{\alpha}_{i}^{2} \mathbf{x}^{*}\right), \\
i=1, \ldots, k .
\end{array}
$$

If the $j$ th constraint of $\operatorname{MINMAX}(\hat{\boldsymbol{\mu}})(25)$ is inactive, that is,

$$
\begin{aligned}
& \mu_{\widetilde{G}_{j}}^{-1}\left(\hat{\mu}_{j}-\lambda^{*}\right)-\left(\mathbf{d}_{j}^{1} \mathbf{x}^{*}-L^{-1}\left(\hat{\mu}_{j}-\lambda^{*}\right) \boldsymbol{\alpha}_{j}^{1} \mathbf{x}^{*}\right) \\
& >T_{j}^{-1}\left(\mu_{\hat{p}_{j}}^{-1}\left(\hat{\mu}_{j}-\lambda^{*}\right)\right) \cdot\left(\mathbf{d}_{j}^{2} \mathbf{x}^{*}-L^{-1}\left(\hat{\mu}_{j}-\lambda^{*}\right) \boldsymbol{\alpha}_{j}^{2} \mathbf{x}^{*}\right), \\
& \Leftrightarrow \mu_{\widetilde{G}_{j}}^{-1}\left(\hat{\mu}_{j}-\lambda^{*}\right)>f_{j}\left(\mathbf{x}^{*}, \hat{\mu}_{j}-\lambda^{*}, \mu_{\hat{p}_{j}}^{-1}\left(\hat{\mu}_{j}-\lambda^{*}\right)\right),
\end{aligned}
$$

we can convert the inactive constraint (35) into the active one by applying the bisection method for the reference membership value $\hat{\mu}_{j} \in\left[\lambda^{*}, \lambda^{*}+1\right]$.

For the optimal solution $\left(\mathbf{x}^{*}, \lambda^{*}\right)$ of $\operatorname{MINMAX}(\hat{\boldsymbol{\mu}})(25)$, where the active conditions (34) are satisfied, we solve the $M_{P F}$-Pareto optimality test problem defined as follows:

[ $M_{P F}$-Pareto optimality test problem]

$$
\begin{array}{ll}
\underset{\mathbf{x} \in X, \epsilon_{i} \geq 0, i=1, \ldots, k}{\operatorname{maximize}} & w=\sum_{i=1}^{k} \epsilon_{i}, \\
\text { subject to } \quad T_{i}^{-1}\left(\mu_{\hat{p}_{i}}^{-1}\left(\hat{\mu}_{i}-\lambda^{*}\right)\right) & \\
\cdot & \left(\mathbf{d}_{i}^{2} \mathbf{x}-L^{-1}\left(\hat{\mu}_{i}-\lambda^{*}\right) \boldsymbol{\alpha}_{i}^{2} \mathbf{x}\right) \\
& +\left(\mathbf{d}_{i}^{1} \mathbf{x}-L^{-1}\left(\hat{\mu}_{i}-\lambda^{*}\right) \boldsymbol{\alpha}_{i}^{1} \mathbf{x}\right), \\
& +\epsilon_{i} T_{i}^{-1}\left(\mu_{\hat{p}_{i}}^{-1}\left(\hat{\mu}_{i}-\lambda^{*}\right)\right) \\
& \cdot\left(\mathbf{d}_{i}^{2} \mathbf{x}^{*}-L^{-1}\left(\hat{\mu}_{i}-\lambda^{*}\right) \boldsymbol{\alpha}_{i}^{2} \mathbf{x}^{*}\right) \\
& +\left(\mathbf{d}_{i}^{1} \mathbf{x}^{*}-L^{-1}\left(\hat{\mu}_{i}-\lambda^{*}\right) \boldsymbol{\alpha}_{i}^{1} \mathbf{x}^{*}\right), \\
& i=1, \ldots, k .
\end{array}
$$

For the optimal solution of the above test problem, the following theorem holds.

Theorem 4. For the optimal solution $\check{\mathbf{x}}, \check{\epsilon}_{i}, i=1, \ldots, k$ of the test problem (36), if $w=0$ (equivalently, $\check{\epsilon}_{i}=0, i=1, \ldots, k$ ), $\mathbf{x}^{*} \in X, \mu_{\hat{p}_{i}}^{-1}\left(\hat{\mu}_{i}-\lambda^{*}\right) \in P_{i}, \hat{\mu}_{i}-\lambda^{*} \in H_{i}\left(P_{i}\right), i=1, \ldots, k$ is an $M_{P F}$-Pareto optimal solution.

Proof. From the active conditions (34), it holds that

$$
\hat{\mu}_{i}-\lambda^{*}=\mu_{\widetilde{G}_{i}}\left(f_{i}\left(\mathbf{x}^{*}, \hat{\mu}_{i}-\lambda^{*}, \mu_{\hat{p}_{i}}^{-1}\left(\hat{\mu}_{i}-\lambda^{*}\right)\right)\right),
$$


and it is obvious that $\hat{\mu}_{i}-\lambda^{*}=\mu_{\hat{p}_{i}}\left(\mu_{\hat{p}_{i}}^{-1}\left(\hat{\mu}_{i}-\lambda^{*}\right)\right), i=1, \ldots, k$. Assume that $\mathbf{x}^{*} \in X, \mu_{\hat{p}_{i}}^{-1}\left(\hat{\mu}_{i}-\lambda^{*}\right) \in P_{i}, \hat{\mu}_{i}-\lambda^{*} \in$ $H_{i}\left(P_{i}\right), i=1, \ldots, k$ is not an $M_{P F}$-Pareto optimal solution. Then, there exists some $\mathbf{x} \in X, \hat{p}_{i} \in P_{i}, h_{i} \in H_{i}\left(P_{i}\right), i=$ $1, \ldots, k$ such that

$$
\begin{aligned}
\mu_{D_{G_{i}}}\left(\mathbf{x}, h_{i}, \hat{p}_{i}\right) & \\
=\min \left\{\mu_{\hat{p}_{i}}\left(\hat{p}_{i}\right), \mu_{\widetilde{G}_{i}}\left(f_{i}\left(\mathbf{x}, h_{i}, \hat{p}_{i}\right)\right)\right\} & \\
\geq \mu_{D_{G_{i}}}\left(\mathbf{x}^{*}, \hat{\mu}_{i}-\lambda^{*}, \mu_{\hat{p}_{i}}^{-1}\left(\hat{\mu}_{i}-\lambda^{*}\right)\right) & =\hat{\mu}_{i}-\lambda^{*}, \\
& i=1, \ldots, k,
\end{aligned}
$$

with strict inequality holding for at least one $i$, and $\mu_{\tilde{G}_{i}}\left(f_{i}\left(\mathbf{x}, h_{i}, \hat{p}_{i}\right)\right)=h_{i}, i=1, \ldots, k$. This means that the following inequalities hold:

$$
\begin{gathered}
\mu_{\hat{p}_{i}}\left(\hat{p}_{i}\right) \geq \hat{\mu}_{i}-\lambda^{*}, \quad i=1, \ldots, k, \\
\mu_{\widetilde{G}_{i}}\left(f_{i}\left(\mathbf{x}, h_{i}, \hat{p}_{i}\right)\right) \geq \hat{\mu}_{i}-\lambda^{*}, \quad i=1, \ldots, k .
\end{gathered}
$$

Because of Assumption 1, (8), and $L^{-1}\left(h_{i}\right) \leq L^{-1}\left(\hat{\mu}_{i}-\lambda^{*}\right)$, (39) can be transformed into the following forms

$$
\begin{aligned}
\hat{p}_{i} & \geq \mu_{\hat{p}_{i}}^{-1}\left(\hat{\mu}_{i}-\lambda^{*}\right), \quad i=1, \ldots, k, \\
\hat{p}_{i} & \leq T_{i}\left(\frac{\mu_{\widetilde{G}_{i}}^{-1}\left(\hat{\mu}_{i}-\lambda^{*}\right)-\left(\mathbf{d}_{i}^{1} \mathbf{x}-L^{-1}\left(h_{i}\right) \boldsymbol{\alpha}_{i}^{1} \mathbf{x}\right)}{\mathbf{d}_{i}^{2} \mathbf{x}-L^{-1}\left(h_{i}\right) \boldsymbol{\alpha}_{i}^{2} \mathbf{x}}\right) \\
& \leq T_{i}\left(\frac{\mu_{\widetilde{G}_{i}}^{-1}\left(\hat{\mu}_{i}-\lambda^{*}\right)-\left(\mathbf{d}_{i}^{1} \mathbf{x}-L^{-1}\left(\hat{\mu}_{i}-\lambda^{*}\right) \boldsymbol{\alpha}_{i}^{1} \mathbf{x}\right)}{\mathbf{d}_{i}^{2} \mathbf{x}-L^{-1}\left(\hat{\mu}_{i}-\lambda^{*}\right) \boldsymbol{\alpha}_{i}^{2} \mathbf{x}}\right) \\
i=1, \ldots, k . &
\end{aligned}
$$

From (37), there exists some $\mathbf{x} \in X$ such that

$$
\begin{aligned}
& \mu_{\widetilde{G}_{i}}^{-1}\left(\hat{\mu}_{i}-\lambda^{*}\right)-\left(\mathbf{d}_{i}^{1} \mathbf{x}-L^{-1}\left(\hat{\mu}_{i}-\lambda^{*}\right) \boldsymbol{\alpha}_{i}^{1} \mathbf{x}\right) \\
& \geq T_{i}^{-1}\left(\mu_{\hat{p}_{i}}^{-1}\left(\hat{\mu}_{i}-\lambda^{*}\right)\right) \cdot\left(\mathbf{d}_{i}^{2} \mathbf{x}-L^{-1}\left(\hat{\mu}_{i}-\lambda^{*}\right) \boldsymbol{\alpha}_{i}^{2} \mathbf{x}\right), \\
& \Longleftrightarrow T_{i}^{-1}\left(\mu_{\hat{p}_{i}}^{-1}\left(\hat{\mu}_{i}-\lambda^{*}\right)\right) \cdot\left(\mathbf{d}_{i}^{2} \mathbf{x}^{*}-L^{-1}\left(\hat{\mu}_{i}-\lambda^{*}\right) \boldsymbol{\alpha}_{i}^{2} \mathbf{x}^{*}\right) \\
&+\left(\mathbf{d}_{i}^{1} \mathbf{x}^{*}-L^{-1}\left(\hat{\mu}_{i}-\lambda^{*}\right) \boldsymbol{\alpha}_{i}^{1} \mathbf{x}^{*}\right) \\
& \geq T_{i}^{-1}\left(\mu_{\hat{p}_{i}}^{-1}\left(\hat{\mu}_{i}-\lambda^{*}\right)\right) \cdot\left(\mathbf{d}_{i}^{2} \mathbf{x}-L^{-1}\left(\hat{\mu}_{i}-\lambda\right) \boldsymbol{\alpha}_{i}^{2} \mathbf{x}\right) \\
&+\left(\mathbf{d}_{i}^{1} \mathbf{x}-L^{-1}\left(\hat{\mu}_{i}-\lambda\right) \boldsymbol{\alpha}_{i}^{1} \mathbf{x}\right), \quad i=1, \ldots, k,
\end{aligned}
$$

with strict inequality holding for at least one $i$. This contradicts the fact that $w=0$.

\section{Interactive Algorithm}

Following the above discussions, we are now ready to present an interactive algorithm in order to derive a satisfactory solution from among an $M_{P F}$-Pareto optimal solution set.

[Interactive algorithm].
Step 1. Calculate $\min _{\mathbf{x} \in X} E\left[\overline{\mathbf{d}}_{i}\right] \mathbf{x}, i=1, \ldots, k$, where $\overline{\mathbf{d}}_{i}(\omega) \mathbf{x}$ are central values of LR fuzzy numbers $\widetilde{\mathbf{c}}_{i}(\omega) \mathbf{x}$. Let us denote $\mathbf{x}_{i}$ as the optimal solution of the optimization problem $\min _{\mathbf{x} \in X} E\left[\overline{\mathbf{d}}_{i}\right] \mathbf{x}$. Then, define $f_{i \text { min }} \stackrel{\text { def }}{=} E\left[\overline{\mathbf{d}}_{i}\right] \mathbf{x}_{i}$, and $f_{i \max } \stackrel{\text { def }}{=}$ $\max _{j=1, \ldots, k, j \neq i} E\left[\overline{\mathbf{d}}_{i}\right] \mathbf{x}_{j}$. The decision maker determines the membership functions $\mu_{\widetilde{G}_{i}}(y), i=1, \ldots, k$ in his/her subjective manner, where $\mu_{\widetilde{G}_{i}}\left(f_{i \text { min }}\right)=1, \mu_{\widetilde{G}_{i}}\left(f_{i \max }\right)=0$.

Step 2. The decision maker specifies the interval $P_{i}=$ $\left[p_{i \text { min }}, p_{i \max }\right], i=1, \ldots, k$, where $p_{i \text { min }}$ is an unacceptable maximum value of $\hat{p}_{i}$ and $p_{i \max }$ is a sufficiently satisfactory minimum value of $\hat{p}_{i}$. For the interval $P_{i}$, the decision maker determines the membership function $\mu_{\hat{p}_{i}}\left(\hat{p}_{i}\right)$ according to Assumption 1.

Step 3. Set the initial reference membership values as $\hat{\mu}_{i}=$ $1, i=1, \ldots, k$.

Step 4. Solve MINMAX $(\hat{\boldsymbol{\mu}})$ by combined use of the bisection method $\lambda \in \Lambda$ and the first-phase of the two-phase simplex method of linear programming, and obtain the optimal solution $\left(\mathbf{x}^{*}, \lambda^{*}\right)$. For the optimal solution $\left(\mathbf{x}^{*}, \lambda^{*}\right)$, the corresponding $M_{P F}$-Pareto optimality test problem (36) is formulated and solved.

Step 5. If the decision maker is satisfied with the current values of the $M_{P F}$-Pareto optimal solution $\mu_{D_{G_{i}}}\left(\mathbf{x}^{*}, h_{i}^{*}, \hat{p}_{i}^{*}\right), i=$ $1, \ldots, k$, where $\hat{p}_{i}^{*}=\mu_{\hat{p}_{i}}^{-1}\left(\hat{\mu}_{i}-\lambda^{*}\right), h_{i}^{*}=\hat{\mu}_{i}-\lambda^{*}, i=$ $1, \ldots, k$, then stop. Otherwise, the decision maker updates the reference membership values $\hat{\mu}_{i}, i=1, \ldots, k$, and returns to Step 4.

\section{Numerical Example}

In order to demonstrate the feasibility of the proposed method, consider the following two-objective fuzzy random linear programming problem $[11,12]$ :

$$
\begin{array}{ll}
\text { minimize } & \tilde{\overline{\mathbf{c}}}_{1} \mathbf{x}=\tilde{\bar{c}}_{11} x_{1}+\tilde{\bar{c}}_{12} x_{2}+\tilde{\bar{c}}_{13} x_{3}, \\
\text { minimize } & \tilde{\overline{\mathbf{c}}}_{2} \mathbf{x}=\tilde{\bar{c}}_{21} x_{1}+\tilde{\bar{c}}_{22} x_{2}+\tilde{\bar{c}}_{23} x_{3}, \\
\text { subject to } & 2 x_{1}+6 x_{2}+3 x_{3} \leq 150, \\
& 6 x_{1}+3 x_{2}+5 x_{3} \leq 175, \\
& 5 x_{1}+4 x_{2}+2 x_{3} \leq 160 \\
& 2 x_{1}+2 x_{2}+3 x_{3} \geq 90, \\
& x_{i} \geq 0, \quad i=1,2,3 .
\end{array}
$$

It is assumed here that a realization $\widetilde{\bar{c}}_{i j}(\omega)$ of an LR-type fuzzy random variable $\tilde{\widetilde{c}}_{i j}$ is an LR fuzzy number whose membership function is defined as follows:

$$
\mu_{\tilde{c} i j}(s)= \begin{cases}L\left(\frac{d_{i j}^{1}+\bar{t}_{i}(\omega) d_{i j}^{2}-s}{\alpha_{i j}^{1}+\bar{t}_{i}(\omega) \alpha_{i j}^{2}}\right) & \left(s \leq \bar{d}_{i j}(\omega)\right), \\ R\left(\frac{s-d_{i j}^{1}+\bar{t}_{i}(\omega) d_{i j}^{2}}{\beta_{i j}^{1}+\bar{t}_{i}(\omega) \beta_{i j}^{2}}\right) & \left(s>\bar{d}_{i j}(\omega)\right),\end{cases}
$$


TABLE 1: Parameter values for LR-type fuzzy random variables $\widetilde{\bar{c}}_{i j}$.

\begin{tabular}{lccc}
\hline$j$ & 1 & 2 & 3 \\
\hline$d_{1 j}^{1}$ & 2 & 1 & 3 \\
$d_{2 j}^{1}$ & -7 & -7 & -9 \\
$\alpha_{1 j}^{1}$ & 0.5 & 0.4 & 0.5 \\
$\alpha_{2 j}^{1}$ & 0.3 & 0.5 & 0.4 \\
$\beta_{1 j}^{1}$ & 0.6 & 0.5 & 0.6 \\
$\beta_{2 j}^{1}$ & 0.4 & 0.5 & 0.5 \\
$d_{1 j}^{2}$ & 1.3 & 1.1 & 1.2 \\
$d_{2 j}^{2}$ & 1.1 & 1.2 & 1.1 \\
$\alpha_{1 j}^{2}$ & 0.05 & 0.04 & 0.05 \\
$\alpha_{2 j}^{2}$ & 0.05 & 0.04 & 0.05 \\
$\beta_{1 j}^{2}$ & 0.06 & 0.05 & 0.06 \\
$\beta_{2 j}^{2}$ & 0.06 & 0.06 & 0.05 \\
\hline
\end{tabular}

TABLE 2: Interactive processes.

\begin{tabular}{lccc}
\hline & 1 & 2 & 3 \\
\hline$\hat{\mu}_{1}$ & 1 & 0.5 & 0.52 \\
$\hat{\mu}_{2}$ & 1 & 0.6 & 0.59 \\
\hline$\mu_{D_{G_{1}}}\left(\mathbf{x}^{*}, h_{1}^{*}, \hat{p}_{1}^{*}\right)$ & 0.564271 & 0.514421 & 0.529412 \\
$\mu_{D_{G_{2}}}\left(\mathbf{x}^{*}, h_{2}^{*}, \hat{p}_{2}^{*}\right)$ & 0.564271 & 0.614421 & 0.599412 \\
\hline$\hat{p}_{1}^{*}$ & 0.578193 & 0.562545 & 0.567250 \\
$\hat{p}_{2}^{*}$ & 0.551616 & 0.581684 & 0.572685 \\
\hline$f_{1}\left(\mathbf{x}^{*}, h_{1}^{*}, \hat{p}_{1}^{*}\right)$ & 84.3370 & 85.4053 & 85.0840 \\
$f_{2}\left(\mathbf{x}^{*}, h_{2}^{*}, \hat{p}_{2}^{*}\right)$ & -311.601 & -313.966 & -313.258 \\
\hline
\end{tabular}

where $L(t)=R(t)=\max \{0,1-t\}$, and the parameters $d_{i j}^{1}, d_{i j}^{2}, \alpha_{i j}^{1}, \alpha_{i j}^{2}, \beta_{i j}^{1}, \beta_{i j}^{2}$ are given in Table $1[11,12]$.

Moreover, $\bar{t}_{i}, i=1,2$ are Gaussian random variables defined as $\bar{t}_{i} \sim N(0,1)$.

At Step 1, calculate $\min _{\mathbf{x} \in X} E\left[\overline{\mathbf{d}}_{i}\right] \mathbf{x}, i=1,2$, and we obtain $f_{1 \text { min }}=75, f_{1 \max }=96.42857$ and $f_{2 \min }=$ $-332.143, f_{2 \max }=-285$. We assume that the decision maker adopts the linear membership functions $\mu_{\widetilde{G}_{i}}(y), i=1, \ldots, k$ defined as follows:

$$
\mu_{\widetilde{G}_{i}}\left(f_{i}\left(\mathbf{x}, h_{i}, \hat{p}_{i}\right)\right)=\frac{f_{i \max }-f_{i}\left(\mathbf{x}, h_{i}, \hat{p}_{i}\right)}{f_{i \max }-f_{i \min }}, \quad i=1,2
$$

At Step 2, the decision maker specifies the intervals $P_{1}=\left[p_{1 \min }, p_{1 \max }\right]=[0.401066,0.714968]$ and $P_{2}=$ $\left[p_{2 \min }, p_{2 \max }\right]=[0.213304,0.812859]$. Then, we assume that the decision maker adopts the linear membership functions $\mu_{\hat{p}_{i}}\left(\hat{p}_{i}\right)$ defined as follows:

$$
\mu_{\hat{p}_{i}}\left(\hat{p}_{i}\right)=\frac{\hat{p}_{i}-p_{i \min }}{\left(p_{i \max }-p_{i \min }\right)}, \quad i=1,2
$$

At Step 3, set the initial reference membership values as $\left(\hat{\mu}_{1}, \hat{\mu}_{2}\right)=(1,1)$. Solve $\operatorname{MINMAX}(\hat{\boldsymbol{\mu}})$ by combined use of the bisection method with respect to $\lambda$ and the first-phase of the two-phase simplex method of linear programming to obtain the corresponding $M_{P F}$-Pareto optimal solution $\left(\mathbf{x}^{*}, \lambda^{*}\right)$ (Step 4). Consider

$$
\begin{aligned}
& \mu_{\widetilde{G}_{1}}\left(f_{1}\left(\mathbf{x}^{*}, h_{1}^{*}, \hat{p}_{1}^{*}\right)\right)=\mu_{\hat{p}_{1}}\left(\hat{p}_{1}^{*}\right)=0.564271, \\
& \mu_{\widetilde{G}_{2}}\left(f_{2}\left(\mathbf{x}^{*}, h_{2}^{*}, \hat{p}_{2}^{*}\right)\right)=\mu_{\hat{p}_{2}}\left(\hat{p}_{2}^{*}\right)=0.564271 .
\end{aligned}
$$

The hypothetical decision maker is not satisfied with the current value of the $M_{P F}$-Pareto optimal solution $\left(\mathbf{x}^{*}, \lambda^{*}\right)$, and, in order to improve $\mu_{D_{G_{2}}}(\cdot)=\min \left\{\mu_{\tilde{G}_{2}}(\cdot), \mu_{\hat{p}_{2}}(\cdot)\right\}$ at the expense of $\mu_{D_{G_{1}}}(\cdot)=\min \left\{\mu_{\widetilde{G}_{1}}(\cdot), \mu_{\hat{p}_{1}}(\cdot)\right\}$, he/she updates his/her reference membership values as $\left(\hat{\mu}_{1}, \hat{\mu}_{2}\right)=(0.5,0.6)$ (Step 5). Then, the corresponding $M_{P F}$-Pareto optimal solution is obtained by solving $\operatorname{MINMAX}(\hat{\boldsymbol{\mu}})$ (Step 4). Consider

$$
\begin{aligned}
& \mu_{\widetilde{G}_{1}}\left(f_{1}\left(\mathbf{x}^{*}, h_{1}^{*}, \hat{p}_{1}^{*}\right)\right)=\mu_{\hat{p}_{1}}\left(\hat{p}_{1}^{*}\right)=0.514421, \\
& \mu_{\widetilde{G}_{2}}\left(f_{2}\left(\mathbf{x}^{*}, h_{2}^{*}, \hat{p}_{2}^{*}\right)\right)=\mu_{\hat{p}_{2}}\left(\hat{p}_{2}^{*}\right)=0.614421 .
\end{aligned}
$$

For the current value of the $M_{P F}$-Pareto optimal solution, the hypothetical decision maker updates his/her reference membership values $\left(\hat{\mu}_{1}, \hat{\mu}_{2}\right)=(0.52,0.59)$ in order to improve $\mu_{D_{G_{1}}}(\cdot)$ at the expense of $\mu_{D_{G_{2}}}(\cdot)$ slightly (Step 5). The corresponding $M_{P F}$-Pareto optimal solution is obtained by solving MINMAX $(\hat{\boldsymbol{\mu}})$ (Step 4). Consider

$$
\begin{gathered}
\mu_{\widetilde{G}_{1}}\left(f_{1}\left(\mathbf{x}^{*}, h_{1}^{*}, \hat{p}_{1}^{*}\right)\right)=\mu_{\hat{p}_{1}}\left(\hat{p}_{1}^{*}\right)=0.529412, \\
\mu_{\widetilde{G}_{2}}\left(f_{2}\left(\mathbf{x}^{*}, h_{2}^{*}, \hat{p}_{2}^{*}\right)\right)=\mu_{\hat{p}_{2}}\left(\hat{p}_{2}^{*}\right)=0.599412 .
\end{gathered}
$$

Then, since the hypothetical decision maker is satisfied with the current value of the $M_{P F}$-Pareto optimal solution, stop the interactive processes (Step 5). The interactive processes under the hypothetical decision maker are summarized in Table 2.

In order to compare our proposed approach with the previous ones, let us consider multiobjective programming problem $\operatorname{MOP}(\hat{\mathbf{p}})(10)$, which is formulated through a fractile optimization model. We obtain one of the Pareto optimal solutions of $\operatorname{MOP}(\widehat{\mathbf{p}})(10)$, which are defined in membership space, that is, $\mu_{\widetilde{G}_{i}}\left(f_{i}\left(\mathbf{x}, h_{i}, \hat{p}_{i}\right)\right), i=1, \ldots, k$. Similar to $\operatorname{MINMAX}(\hat{\boldsymbol{\mu}})$ (19), we can formulate the following minmax problem to obtain the Pareto optimal solution of $\operatorname{MOP}(\hat{\mathbf{p}})(10)$, where $\hat{p}_{i}, i=1,2$ are not decision variables but constant values:

$[\operatorname{MINMAX}(\hat{\mathbf{p}}, \hat{\boldsymbol{\mu}})]$

$$
\begin{aligned}
& \min _{\mathbf{x} \in X, h_{i} \in[0,1], i=1, \ldots, k, \lambda \in \Lambda} \lambda, \\
& \text { subject to } \quad \hat{\mu}_{i}-\mu_{\widetilde{G}_{i}}\left(f_{i}\left(\mathbf{x}, h_{i}, \hat{p}_{i}\right)\right) \leq \lambda, \quad i=1, \ldots, k, \\
& \\
& \mu_{\widetilde{G}_{i}}\left(f_{i}\left(\mathbf{x}, h_{i}, \hat{p}_{i}\right)\right)=h_{i}, \quad i=1, \ldots, k .
\end{aligned}
$$

In $\operatorname{MINMAX}(\hat{\mathbf{p}}, \hat{\boldsymbol{\mu}})$, we assume that the decision maker sets his/her permissible probability levels as $\hat{p}_{1}=\hat{p}_{2}=0.75$, and the reference membership values as $\hat{\mu}_{1}=\hat{\mu}_{2}=1$. Then, the corresponding Pareto optimal solution is obtained as follows:

$$
\begin{gathered}
f_{1}\left(\mathbf{x}^{*}, h_{1}^{*}, 0.75\right)=94.0338 \\
f_{2}\left(\mathbf{x}^{*}, h_{2}^{*}, 0.75\right)=-290.269 \\
\mu_{\widetilde{G}_{i}}\left(f_{i}\left(\mathbf{x}^{*}, h_{i}^{*}, 0.75\right)\right)=0.11176, \quad \hat{p}_{i}=0.75, \quad i=1,2 .
\end{gathered}
$$


In our proposed algorithm, by solving $\operatorname{MINMAX}(\hat{\boldsymbol{\mu}})$ (25) for the reference membership values $\hat{\mu}_{1}=\hat{\mu}_{2}=1$, the $M_{P F^{-}}$ Pareto optimal solution is obtained as follows (see the first iteration of Table 2):

$$
\begin{gathered}
f_{1}\left(\mathbf{x}^{*}, h_{1}^{*}, \hat{p}_{1}^{*}\right)=84.3370, \quad f_{2}\left(\mathbf{x}^{*}, h_{2}^{*}, \hat{p}_{2}^{*}\right)=-311.601, \\
\mu_{D_{G_{i}}}\left(\mathbf{x}^{*}, h_{i}^{*}, \hat{p}_{i}^{*}\right)=0.564271, \quad i=1,2, \\
\hat{p}_{1}^{*}=0.578193, \quad \hat{p}_{2}^{*}=0.551616 .
\end{gathered}
$$

This means that a proper balance between permissible probability levels and the corresponding objective functions in a fractile optimization model is attained in membership space.

\section{Conclusion}

In this paper, we have proposed an interactive fuzzy decision making method for multiobjective fuzzy random linear programming problems to obtain a satisfactory solution from among an extended Pareto optimal solution set. In the proposed method, the decision maker is required to specify the membership functions for the fuzzy goals of not only objective functions but also the permissible probability levels. An extended Pareto optimal concept called $\mathrm{M}_{\mathrm{PF}^{-}}$ Pareto optimal was introduced. The satisfactory solution can be obtained by updating the reference membership values and solving the corresponding minmax problem based on the linear programming technique. An illustrative numerical example demonstrated the feasibility of the proposed method. In the near future, applications of the proposed method to the real-world decision making situations as well as extensions to more general cases will be required.

\section{References}

[1] J. R. Birge and F. Louveaux, Introduction to Stochastic Programming, Springer, London, UK, 1997.

[2] A. Charnes and W. W. Cooper, "Chance-constrained programming," Management Science, vol. 6, pp. 73-79, 1959.

[3] G. B. Dantzig, "Linear programming under uncertainty," Management Science, vol. 1, pp. 197-206, 1955.

[4] P. Kall and J. Mayer, Stochastic Linear Programming Models, Theory, and Computation, Springer, New York, NY, USA, 2005.

[5] Y.-J. Lai and C. L. Hwang, Fuzzy Mathematical Programming, vol. 394 of Lecture Notes in Economics and Mathematical Systems, Springer, Berlin, Germany, 1992.

[6] M. Sakawa, Fuzzy Sets and Interactive Multiobjective Optimization, Applied Information Technology, Plenum Press, New York, NY, USA, 1993.

[7] H. J. Zimmermann, Fuzzy Sets, Decision-Making and Expert Systems, KluwerAcademic Publishers, Boston, Mass, USA, 1987.

[8] H. Kwakernaak, "Fuzzy random variables-I," Information Sciences, vol. 15, no. 1, pp. 1-29, 1978.

[9] M. K. Luhandjula and M. M. Gupta, "On fuzzy stochastic optimization," Fuzzy Sets and Systems, vol. 81, no. 1, pp. 4755, 1996.

[10] W. Guang-Yuan and Q. Zhong, "Linear programming with fuzzy random variable coefficients," Fuzzy Sets and Systems, vol. 57, no. 3, pp. 295-311, 1993.
[11] M. Sakawa, I. Nishizaki, and H. Katagiri, Fuzzy Stochastic Multiobjective Programming, Springer, New York, NY, USA, 2011.

[12] H. Katagiri, M. Sakawa, K. Kato, and I. Nishizaki, "Interactive multiobjective fuzzy random linear programming: maximization of possibility and probability," European Journal of Operational Research, vol. 188, no. 2, pp. 530-539, 2008.

[13] H. Katagiri and M. Sakawa, "Interactive multiobjective fuzzy random programming through the level set-based probability model," Information Sciences, vol. 181, no. 9, pp. 1641-1650, 2011.

[14] H. Yano and K. Matsui, "Fuzzy approaches for multiobjective fuzzy random linear programming problems through a probability maximization model," in Proceedings of the International MultiConference of Engineers and Computer Scientists, (IMECS '11), pp. 1349-1354, Hong Kong, March 2011.

[15] J. J. Glen, "Mathematical models in farm planning: a survey," Operations Research, vol. 35, no. 5, pp. 641-666, 1987.

[16] K. Hayashi, "Multicriteria analysis for agricultural resource management: a critical survey and future perspectives," European Journal of Operational Research, vol. 122, no. 2, pp. 486$500,2000$.

[17] T. Itoh, H. Ishii, and T. Nanseki, "A model of crop planning under uncertainty in agricultural management," International Journal of Production Economics, vol. 81-82, pp. 555-558, 2003.

[18] T. Toyonaga, T. Itoh, and H. Ishii, "A crop planning problem with fuzzy random profit coefficients," Fuzzy Optimization and Decision Making, vol. 4, no. 1, pp. 51-69, 2005.

[19] D. Dubois and H. Prade, Fuzzy Sets and Systems, vol. 144 of Mathematics in Science and Engineering, Academic Press, New York, NY, USA, 1980.

[20] M. L. Puri and D. A. Ralescu, "Fuzzy random variables," Journal of Mathematical Analysis and Applications, vol. 114, no. 2, pp. 409-422, 1986.

[21] H. Katagiri, M. Sakawa, K. Kato, and S. Ohsaki, "An interactive fuzzy satisficing method for fuzzy random multiobjective linear programming problems through the fractile optimization model using possibility and necessity measures," in Proceedings of the 9th Asia Pacific Management Conference, pp. 795-802, 2003. 

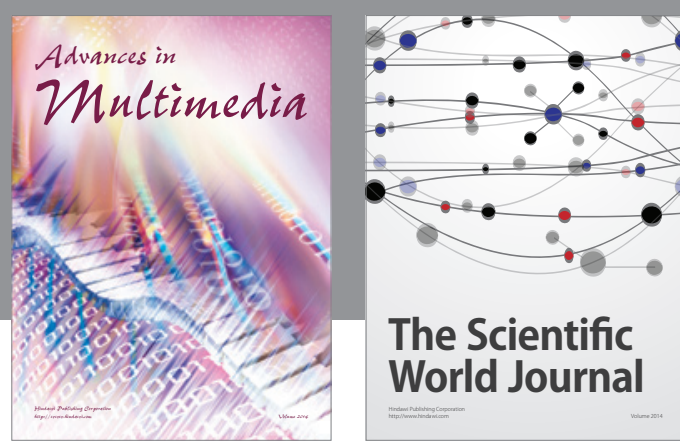

The Scientific World Journal
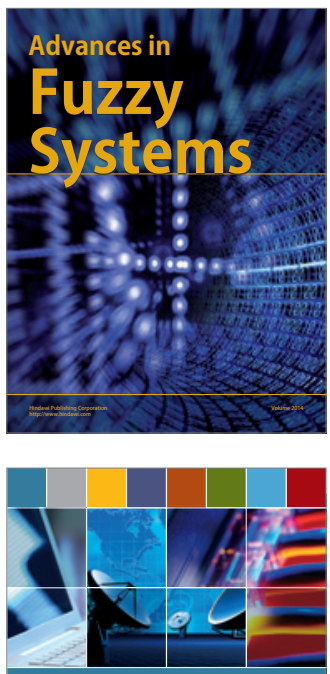

Computer Networks and Communications
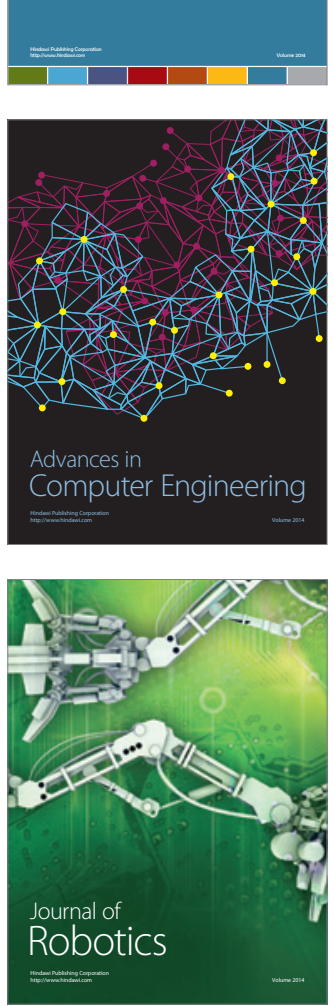
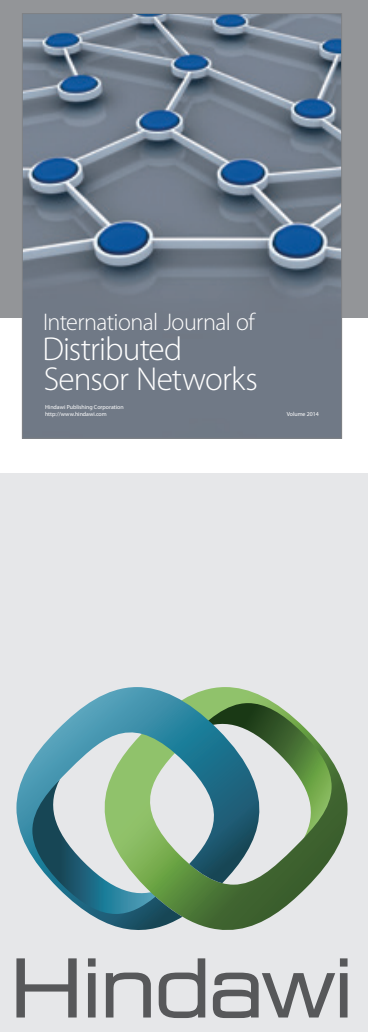

Submit your manuscripts at

http://www.hindawi.com
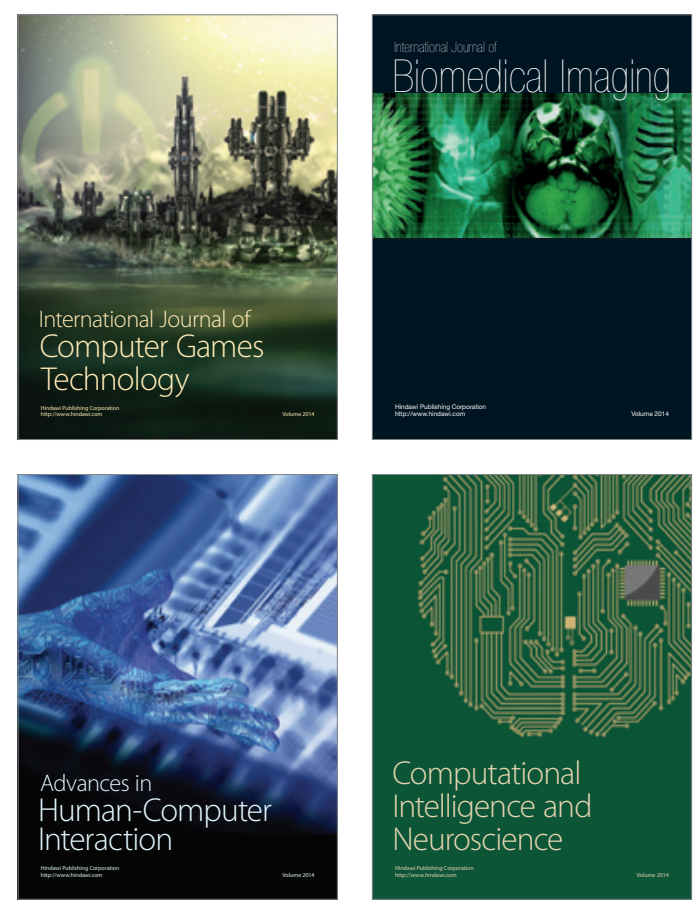
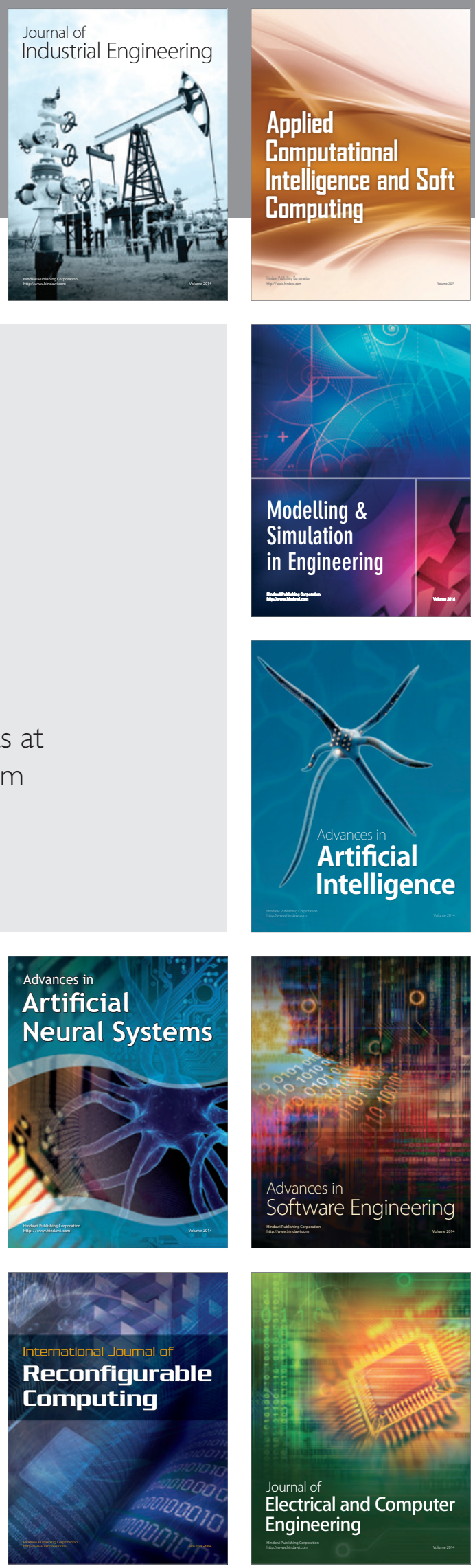